\title{
Geodesic locally starshaped G-invex sets and geodesic semilocal preinvex functions
}

\author{
Sandeep Kumar Porwal \\ Department of Mathematics \\ CSJM University Campus, Kanpur, U.P., India \\ Email: skpmathsdstcims@gmail.com
}

\begin{abstract}
In this paper, we introduce a new class of sets and functions, namely geodesic locally starshaped $G$-invex sets and geodesic semilocal preinvex functions on Riemannian manifolds. We study the properties of these classes of sets and functions and derive certain characterizations. The results of the paper extend and unify several known results from literature to a more general class of functions as well as in more general space setting.
\end{abstract}

MSC: 52A01, 90C26, 90C30, 53B21

Keywords Locally starshaped invex set, geodesic semilocal preinvex functions, semistrictly geodesic semilocal preinvex function, geodesic locally starshaped $G$-invex set.

\section{Introduction}

Convexity plays an important role in various fields like management science, engineering, mathematical economics, optimization theory and Riemannian manifolds etc. However, the notion of convexity does no longer suffice in several real world applications. Therefore, it is essential to consider a large class of generalized convex functions and also look for practical criteria of convexity. For recent development and survey, we refer to Mishra and Upadhyay [17, 18, 19, 20, 21] and Mishra et al. [22].

Mangasarian [15] has introduced the notion of pseudoconvexity and pseudoconcavity. The concept of invex function introduced by Hanson [8] and named by Craven [6] is a significant generalization of the notion of convexity. This work inspired a great deal of subsequent works, which has greatly expanded the role and application of invexity in nonlinear optimization and other branches of pure and applied sciences. Ben-Israel and Mond [5] introduced a new generalization of convex sets and convex functions, later, termed as invex sets and preinvex functions, by Weir and Mond [31] and Weir and Jeyakumar [30].

In 1977, Ewing [7] developed a generalized convexity known as semilocal convexity by reducing the width of the line segment, where the concept is applied to provide sufficient optimality conditions in variational and control problems. Generalizations of semilocal convex functions and their properties have been studied by Kaul and Kaur [9, 10] and Kaur [11]. In 1996, Preda et al. [25] established optimality conditions and duality results for nonlinear programming involving semilocal preinvex and related functions. Later these results are ex- 
tended in [26] for a multiple-objective programming problems. These results have many applications.

It is well-known that in linear topological spaces, the notion of convex sets rely on connecting any two points of the space by line segment. In several realworld applications, it is not possible to connect the points through line segment. This led to the idea of the generalization of the classical notion of convex sets. Udriste [28] and Rapcsak [27] proposed a generalization of the convexity notion by replacing the linear spaces by Riemannian manifolds, the line segments by a geodesic segments between any two points, and the convex function by the positiveness of their Hessian. A geodesic on the Riemannian manifolds is a curve, that locally minimizes the arc length. Udriste [28] generalization is based on the fact that many of the properties of convex programs on Euclidean space carry over to the case of a complete Riemannian manifolds. Following Udriste [28], several other generalizations of convex sets and convex functions have been proposed on the Riemannian manifolds. In order to extend the validity of the results to a larger classes of optimization problems, these concepts have been generalized and extended in several directions using novel and innovative techniques. Several authors have studied the properties of generalized convex functions on Riemannian manifolds.

Pini [24] introduced the notion of invex function on Riemannian manifold and Mititelu [23] investigated its generalizations. Barani and Pouryayevali [2] defined the geodesic invex set, geodesic $\eta$-invex function and geodesic $\eta$-preinvex function on Riemannian manifold and discussed the relation between them. In [3] Barani and Pouryayevali introduced generalized invariant monotone vector fields on Riemannian manifolds and discussed their relationship with generalized invexities. Li et al. [14] studied the weak sharp minima for constrained optimization problems on Riemannian manifolds and their characterizations. Recently, Agarwal et al. [1] have introduced the notion of geodesic $G$-invex sets and geodesic $\eta$-preinvex functions and study their properties.

Motivated by the works of Agarwal et al. [1],Upadhyay et al. [29] and Yang and $\mathrm{Li}$ [32], we introduce the classes of geodesic semilocal preinvex and semilocal semistrictly geodesic preinvex functions on Reimannian manifolds. We establish that under geodesic semilocal preinvexity assumption, a local solution of a minimization problem becomes a golbal one. Moreover, the epigraph of a geodesic semilocal preinvex function is a geodesic semilocal starshaped invex set. Our results extend and unify several known results in the literature such as Agarwal et al. [1], Yang and $\mathrm{Li}$ [32] and references therein to a more genral class of functions as well as to a more general space setting.

\section{Preliminaries and definitions}

In this section, we gave some preliminary notations, about Riemannian manifolds and some basic definitions which will be used throughout this paper. For preliminary part of this section we refer to [2].

Definition 2.1 [16] A subset $S \subseteq \mathbb{R}^{n}$ is said to be locally starshaped invex set with respect to $\eta$ if for any $x, y \in S$, there exist a positive maximal number $0<a_{\eta}(x, y) \leq 1$ such that

$$
y+\lambda \eta(x, y) \in S, \quad \forall \lambda \in\left[0, a_{\eta}(x, y)\right] .
$$


Definition 2.2 Let $M$ be a Riemannian manifold and $\eta: M \times M \rightarrow T M$ be a vectorial function such that for every $x, y \in M, \eta(x, y) \in T_{y} M$. A nonempty subset $S \subseteq M$ is called geodesic locally starshaped invex set with respect to $\eta$, if for any $x, y \in S$, there exist a positive maximal number $0<a_{\eta}(x, y) \leq 1$ and $a$ unique geodesic $\gamma:\left[0, a_{\eta}(x, y)\right] \rightarrow M$, such that

$$
\gamma_{x, y}(0)=y, \gamma_{x, y}^{\prime}(0)=\eta(x, y), \gamma_{x, y}(\lambda) \in S, \quad \forall \lambda \in\left[0, a_{\eta}(x, y)\right] .
$$

For further details on differential and Riemannian geometry, we refer to $[13,12]$.

Definition 2.3 Let $M$ be a Riemannian manifold and $S \subseteq M$ be a geodesic locally starshaped invex set with respect to $\eta: M \times M \rightarrow T M$. We say that a function $f: S \rightarrow \mathbb{R}$ is geodesic semilocal preinvex (gslpi) on $S$ if for any $x, y \in S$, (with a maximal positive $a_{\eta}(x, y) \leq 1$ satisfying (2.1)), there exist a positive number $0<d_{\eta}(x, y) \leq a_{\eta}(x, y)$, such that

$$
f\left(\gamma_{x, y}(\lambda)\right) \leq \lambda f(x)+(1-\lambda) f(y), \quad \forall \lambda \in\left[0, d_{\eta}(x, y)\right] .
$$

Definition 2.4 Let $M$ be a Riemannian manifold and $S \subseteq M$ be a geodesic locally starshaped invex set with respect to $\eta: M \times M \rightarrow T M$. We say that a function $f: S \rightarrow \mathbb{R}$ is strictly geodesic semilocal preinvex (sgslpi) on $S$ if for any $x, y \in S, x \neq y$, (with a maximal positive $a_{\eta}(x, y) \leq 1$ satisfying (2.1)), there exist a positive number $0<d_{\eta}(x, y) \leq a_{\eta}(x, y)$, such that

$$
f\left(\gamma_{x, y}(\lambda)\right)<\lambda f(x)+(1-\lambda) f(y), \quad \forall \lambda \in\left[0, d_{\eta}(x, y)\right] .
$$

Definition 2.5 Let $M$ be a Riemannian manifold and $S \subseteq M$ be a geodesic locally starshaped invex set with respect to $\eta: M \times M \rightarrow T M$. We say that a function $f: S \rightarrow \mathbb{R}$ is semistrictly geodesic semilocal preinvex (ssgslpi) if $\forall x, y \in S, f(x) \neq f(y)$, (with a maximal positive $a_{\eta}(x, y) \leq 1$ satisfying (2.1)), there exist a positive number $0<d_{\eta}(x, y) \leq a_{\eta}(x, y)$, such that

$$
f\left(\gamma_{x, y}(\lambda)\right)<\lambda f(x)+(1-\lambda) f(y), \quad \forall \lambda \in\left[0, d_{\eta}(x, y)\right] .
$$

Definition 2.6 Any set $S \subseteq M \times \mathbb{R}$ is said to be geodesic locally starshaped $G$-invex set if there exist $\eta: M \times M \rightarrow T M$, and a positive maximal number $0<a_{\eta}(x, y) \leq 1$ such that for any pair of $(x, \alpha),(y, \beta) \in S$, we have

$$
\left(\gamma_{x, y}(\lambda), \lambda \alpha+(1-\lambda) \beta\right) \in S, \quad \forall \lambda \in\left[0, a_{\eta}((x, \alpha),(y, \beta))\right] .
$$

\section{Properties of geodesic locally starshaped G- invex sets and geodesic semilocal preinvex func- tions}

In this section, we derive some properties of geodesic semilocal preinvex functions and semistrictly geodesic semilocal preinvex functions.

Theorem 3.1 Let $S \subseteq M$ be a nonempty geodesic locally starshaped invex set with respect to $\eta: M \times M \rightarrow T M$ and $f: S \rightarrow \mathbb{R}$ be a semistrictly geodesic semilocal preinvex function. If $y \in S$ is a local optimal solution to the problem (P)

$$
\operatorname{Min} f(x)
$$




$$
\text { s.t. } x \in S \text {, }
$$

then $y$ is global minimum in $(P)$.

Proof Suppose that $y \in S$ is a local minimum. Then, there is a neighbourhood $N_{\varepsilon}(y)$ such that

$$
f(y) \leq f(x), \forall x \in S \cap N_{\varepsilon}(y) .
$$

If $y$ is not a global minimum of $f$ then there exists a point $x^{*} \in S$ such that

$$
f\left(x^{*}\right)<f(y)
$$

Since $S$ is a geodesic locally starshaped invex set with respect to $\eta$, there exists an unique geodesic $\gamma:\left[0, a_{\eta}\left(x^{*}, y\right)\right] \rightarrow M$ such that

$$
\gamma_{x^{*}, y}(0)=y, \gamma_{x^{*}, y}^{\prime}(0)=\eta\left(x^{*}, y\right), \gamma_{x^{*}, y}(\lambda) \in S, \forall \lambda \in\left[0, a_{\eta}\left(x^{*}, y\right)\right] .
$$

If we choose $\varepsilon>0$ small enough such that $d(\gamma(\lambda), y)<\varepsilon$, then $\gamma(\lambda) \in N_{\varepsilon}(y)$. By the semistrictly geodesic semilocal preinvexity of $f$, there exists $b_{\eta}\left(x^{*}, y\right)<$ $a_{\eta}\left(x^{*}, y\right)$, such that

$$
f(\gamma(\lambda))<\lambda f\left(x^{*}\right)+(1-\lambda) f(y)<f(y), \quad \forall \lambda \in\left[0, b_{\eta}\left(x^{*}, y\right)\right] .
$$

Therefore, for each $\gamma(\lambda) \in S \cap N_{\varepsilon}(y), f(\gamma(\lambda))<f(y)$, which is a contradiction to (3.1). Hence the result follows.

Theorem 3.2 Let $f: S \rightarrow \mathbb{R}$ be a semistrictly geodesic semilocal preinvex function on a geodesic locally starshaped invex set $S \subseteq M$ with respect to $\eta$, and let $g: I \rightarrow \mathbb{R}$ be a convex and strictly increasing function, where range $(f) \subseteq I$. Then, the composite function $g(f)$ is a semistrictly geodesic semilocal preinvex function on $S$.

Proof For any $x, y \in S, \lambda \in\left(0, d_{\eta}(x, y)\right)$, if $g(f(x)) \neq g(f(y))$, then $f(x) \neq f(y)$. Since $f$ is a semistrictly geodesic semilocal preinvex function, we have

$$
f\left(\gamma_{x, y}(\lambda)\right)<\lambda f(x)+(1-\lambda) f(y), \quad \forall \lambda \in\left[0, d_{\eta}(x, y)\right]
$$

From the convexity and strict increasing property of $g$, for all $\lambda \in\left[0, d_{\eta}(x, y)\right]$, it follows that

$$
\begin{aligned}
g\left[f\left(\gamma_{x, y}(\lambda)\right)\right] & <g[\lambda f(x)+(1-\lambda) f(y)] \\
& <\lambda g(f(x))+(1-\lambda) g(f(y)) .
\end{aligned}
$$

Hence, $g(f)$ is a semistrictly geodesic semilocal preinvex function on $S$.

Theorem 3.3 Let $S \subseteq M$ be a geodesic locally starshaped invex set with respect to $\eta$, then $f$ is a geodesic semilocal preinvex on $S$ with respect to $\eta$ if and only if its epigraph $G_{f}=:\{(x, \alpha): x \in S, f(x) \leq \alpha, \alpha \in \mathbb{R}\}$ is a geodesic locally starshaped invex set with respect to $\eta$ corresponding to $M$. 
Proof Assume that $f$ is geodesic semilocal preinvex on $S$ with respect to $\eta$ and $\left(x, \alpha_{1}\right),\left(y, \alpha_{2}\right) \in G_{f}$, then $x, y \in S$, and $f(x) \leq \alpha_{1}, f(y) \leq \alpha_{2}$. Since $S$ is a geodesic locally starshaped invex set, there exists a maximal positive number $0<a_{\eta}(x, y) \leq 1$, such that

$$
\gamma_{x, y}(\lambda) \in S, \quad \forall \lambda \in\left[0, a_{\eta}(x, y)\right]
$$

In addition, in view of $f$ being a geodesic semilocal preinvex function on $S$ with respect to $\eta$, there is a positive number $d_{\eta}(x, y) \leq a_{\eta}(x, y)$ such that

$$
f\left(\gamma_{x, y}(\lambda)\right) \leq \lambda f(x)+(1-\lambda) f(y) \leq \lambda \alpha_{1}+(1-\lambda) \alpha_{2}, \forall \lambda \in\left[0, d_{\eta}(x, y)\right],
$$

i.e.

$$
\left(\gamma_{x, y}(\lambda), \lambda \alpha_{1}+(1-\lambda) \alpha_{2}\right) \in G_{f}, \forall \lambda \in\left[0, d_{\eta}(x, y)\right] .
$$

Therefore, $G_{f}=\{(x, \alpha): x \in S, f(x) \leq \alpha, \alpha \in \mathbb{R}\}$ is a geodesic locally starshaped invex set with respect to $\eta$ corresponding to $M$.

Conversely, if $G_{f}$ is a geodesic locally starshaped invex set with respect to $\eta$ corresponding to $M$, then for any points $(x, f(x)),(y, f(y)) \in G_{f}$, there exists a maximal positive number $0<a_{\eta}((x, f(x)),(y, f(y))) \leq 1$ such that

$$
\left(\gamma_{x, y}(\lambda), \lambda f(x)+(1-\lambda) f(y)\right) \in G_{f}, \quad \forall \lambda \in\left[0, a_{\eta}((x, f(x)),(y, f(y)))\right] .
$$

which implies that

$$
f\left(\gamma_{x, y}(\lambda)\right) \leq \lambda f(x)+(1-\lambda) f(y), \quad \forall \lambda \in\left[0, a_{\eta}((x, f(x)),(y, f(y)))\right] .
$$

Hence, $f$ is a geodesic semilocal preinvex on $S$.

Theorem 3.4 Let $S_{i}$ where $i \in I$, be a family of geodesic locally starshaped $G$-invex sets in $M \times \mathbb{R}$ with respect to the same $\eta: M \times M \rightarrow T M$. Then, their intersection $\bigcap_{i \in I} S_{i}$ is also a geodesic locally starshaped $G$-invex set.

Proof Let $(x, \alpha),(y, \beta) \in \bigcap_{i \in I} S_{i}$. Then, $(x, \alpha),(y, \beta) \in S_{i}$, for each $i \in I$. But $S_{i}, i \in I$ are all geodesic locally starshaped $G$-invex set for each $i \in I$, it follows that

$$
\left(\gamma_{x, y}(\lambda), \lambda \alpha+(1-\lambda) \beta\right) \in S_{i}, \quad \forall \lambda \in\left[0, a_{i}((x, \alpha),(y, \beta))\right], i \in I .
$$

Taking $a((x, \alpha),(y, \beta))=\min a_{i}((x, \alpha),(y, \beta)), i \in I$, we have

$$
\left(\gamma_{x, y}(\lambda), \lambda \alpha+(1-\lambda) \beta\right) \in \bigcap_{i \in I} S_{i}, \quad \forall \lambda \in[0, a((x, \alpha),(y, \beta))] .
$$

Hence, the result follows.

Theorem 3.5 Let $S \subseteq M$ be a geodesic locally starshaped invex set with respect to $\eta: M \times M \rightarrow T M$ and let $f_{i}, i \in I$ be a family of real valued functions, which are geodesic semilocal preinvex for the same $\eta$ and bounded from above on $S$; then function $f(x)=\sup _{i \in I} f_{i}(x)$ is a geodesic semilocal preinvex on $S$. 
Proof Given that each $f_{i}$ is a geodesic semilocal preinvex function for the same $\eta$ on $S$, therefore, from Theorem 3.3, its epigraph

$$
E\left(f_{i}\right)=:\left\{(x, \alpha): x \in S, \alpha \in \mathbb{R}, f_{i}(x) \leq \alpha\right\},
$$

is a geodesic locally starshaped $G$-invex set in $M \times \mathbb{R}$, Therefore, from Theorem 3.4. their intersection

$$
\begin{aligned}
\bigcap_{i \in I} E\left(f_{i}\right) & =\left\{(x, \alpha): x \in S, \alpha \in \mathbb{R}, f_{i}(x) \leq \alpha ; i \in I\right\}, \\
& =\{(x, \alpha): x \in S, \alpha \in \mathbb{R}, f(x) \leq \alpha\},
\end{aligned}
$$

is also a geodesic locally starshaped $G$-invex set in $M \times \mathbb{R}$. It is easy to see that this intersection is the epigraph of $f$. Hence, from Theorem 3.3, the function $f(x)=\sup _{i \in I} f_{i}(x)$ is a geodesic semilocal preinvex on $S$.

Theorem 3.6 Let $S_{i} \subset M,(i=1,2, \ldots, m)$ be a collection of geodesic locally starshaped invex sets with respect to the same $\eta$, then $\bigcap_{i=1}^{m} S_{i}$ is also a geodesic locally starshaped invex set with respect to $\eta$.

Proof For all $x, y \in \bigcap_{i=1}^{m} S_{i}$, we have $x, y \in S_{i}(i=1,2, \ldots, m)$.

Since $S_{i}(i=1,2, \ldots, m)$ are all geodesic locally starshaped invex sets with respect to same $\eta$, then there exist positive numbers $0<a_{i}(x, y) \leq 1(i=$ $1,2, \ldots, m)$ such that

$$
\gamma_{x, y}(\lambda) \in S_{i}, \quad \forall \lambda \in\left[0, a_{i}(x, y)\right], i=1,2, \ldots, m
$$

Taking $a(x, y)=\min a_{i}(x, y), i=1,2, \ldots, m$, we can get

$$
\gamma_{x, y}(\lambda) \in \bigcap_{i=1}^{m} S_{i}, \quad \forall \lambda \in[0, a(x, y)] .
$$

Therefore, the theorem is proved.

Theorem 3.7 If the functions $f_{i}: M \rightarrow \mathbb{R}(i=1,2, \ldots, m)$ are geodesic semilocal preinvex on geodesic locally starshaped invex set $S \subseteq M$ with respect to same $\eta$, then the function

$$
f(x)=\sum_{i=1}^{m} a_{i} f_{i}(x),
$$

is also geodesic semilocal preinvex on $S$ with respect to $\eta$, for all $a_{i} \geq 0, i=$ $1,2, \ldots, m$.

Proof Since $S$ is a geodesic locally starshaped invex set with respect to $\eta$, then for all $x, y \in S$, there exist a positive numbers $0<a(x, y) \leq 1$, such that

$$
\gamma_{x, y}(\lambda) \in S, \quad \forall \lambda \in\left[0, a_{\eta}(x, y)\right]
$$


On the other hand, $f_{i}, i=1,2, \ldots, m$ are all geodesic semilocal preinvex on $S$ with respect to the same $\eta$; thus, there exist positive numbers $d_{i}(x, y) \leq a(x, y)$, such that

$$
f_{i}\left(\gamma_{x, y}(\lambda)\right) \leq \lambda f_{i}(x)+(1-\lambda) f_{i}(y), \forall \lambda \in\left[0, d_{i}(x, y)\right], i=1,2, \ldots, m .
$$

Now, letting $d(x, y)=\min d_{i}(x, y), i=1,2, \ldots, m$, we have

$$
\begin{gathered}
\sum_{i=1}^{m} a_{i} f_{i}\left(\gamma_{x, y}(\lambda)\right) \leq \lambda \sum_{i=1}^{m} a_{i} f_{i}(x)+(1-\lambda) \sum_{i=1}^{m} a_{i} f_{i}(y), \forall \lambda \in[0, d(x, y)] \\
\Rightarrow f\left(\gamma_{x, y}(\lambda)\right) \leq \lambda f(x)+(1-\lambda) f(y), \forall \lambda \in[0, d(x, y)] .
\end{gathered}
$$

That is, $f(x)$ is geodesic semilocal preinvex on $S$ with respect to $\eta$.

Theorem 3.8 If $f$ is a geodesic semilocal preinvex function on a geodesic locally starshaped invex set $S \subseteq M$ with respect to $\eta$, then the lower section of $f$ defined by

$$
S_{\alpha}=\{x \in S: f(x) \leq \alpha\}
$$

is a geodesic locally starshaped invex set for any $\alpha \in \mathbb{R}$.

Proof For any $\alpha \in \mathbb{R}$ and $x, y \in S_{\alpha}$, then $x, y \in S$ and $f(x) \leq \alpha, f(y) \leq \alpha$. Since $S$ is a geodesic locally starshaped invex set, there is a maximal positive number $0<a_{\eta}(x, y) \leq 1$, such that

$$
\gamma_{x, y}(0)=y, \gamma_{x, y}^{\prime}(0)=\eta(x, y), \gamma_{x, y}(\lambda) \in S, \quad \forall \lambda \in\left[0, a_{\eta}(x, y)\right]
$$

In addition, due to the geodesic semilocal preinvexity of $f$, there is a positive number $d_{\eta}(x, y) \leq a(x, y)$, such that

$$
f\left(\gamma_{x, y}(\lambda)\right) \leq \lambda f(x)+(1-\lambda) f(y) \leq \lambda \alpha+(1-\lambda) \alpha=\alpha, \quad \forall \lambda \in\left[0, d_{\eta}(x, y)\right] .
$$

That is,

$$
\gamma_{x, y}(\lambda) \in S_{\alpha}, \quad \forall \lambda \in\left[0, d_{\eta}(x, y)\right] .
$$

Therefore, $S_{\alpha}$ is a geodesic locally starshaped invex set with respect to $\eta$ for any $\alpha \in \mathbb{R}$.

Theorem 3.9 Let $f$ be a real valued function defined on a geodesic locally starshaped invex set $S \subseteq M$, then $f$ is a geodesic semilocal preinvex function with respect to $\eta$ if and only if for each pair of points $x, y \in S$ (with a maximal positive number $0<a_{\eta}(x, y) \leq 1$ satisfying (2.1)) there exists a positive number $d_{\eta}(x, y) \leq a_{\eta}(x, y)$ such that

$$
f\left(\gamma_{x, y}(\lambda)\right)<\lambda \alpha+(1-\lambda) \beta, \quad \forall \lambda \in\left[0, d_{\eta}(x, y)\right]
$$

whenever $f(x)<\alpha, f(y)<\beta$. 
Proof Let $x, y \in S$ and $\alpha, \beta \in \mathbb{R}$ such that $f(x)<\alpha, f(y)<\beta$. Due to the geodesic locally starshaped invexity of $S$, there is a maximal positive number $a_{\eta}(x, y)$, such that

$$
\gamma_{x, y}(\lambda) \in S, \quad \forall \lambda \in\left[0, a_{\eta}(x, y)\right]
$$

In addition, owing to the geodesic semilocal preinvexity of $f$, there is a positive number $d_{\eta}(x, y) \leq a_{\eta}(x, y)$ such that

$$
f\left(\gamma_{x, y}(\lambda)\right) \leq \lambda f(x)+(1-\lambda) f(y)<\lambda \alpha+(1-\lambda) \beta, \quad \forall \lambda \in\left[0, d_{\eta}(x, y)\right] .
$$

Conversely, let $(x, \alpha),(y, \beta) \in G_{f}$ (see epigraph $G_{f}$ in Theorem 3.3), then $x, y \in S, f(x) \leq \alpha$, and $f(y) \leq \beta$. Hence, $f(x)<\alpha+\varepsilon$ and $f(y)<\beta+\varepsilon$ hold for any $\varepsilon>0$. According to the hypothesis, for $x, y \in S$ (with a positive number $0<a_{\eta}(x, y) \leq 1$ satisfying (2.1)), there exists a positive number $d_{\eta}(x, y) \leq$ $a_{\eta}(x, y)$ such that

$$
f\left(\gamma_{x, y}(\lambda)\right)<\lambda \alpha+(1-\lambda) \beta+\varepsilon, \quad \forall \lambda \in\left[0, d_{\eta}(x, y)\right] .
$$

Let $\varepsilon \rightarrow 0^{+}$, then

$$
f\left(\gamma_{x, y}(\lambda)\right) \leq \lambda \alpha+(1-\lambda) \beta, \quad \forall \lambda \in\left[0, d_{\eta}(x, y)\right] .
$$

That is,

$$
\left(\gamma_{x, y}(\lambda), \lambda \alpha+(1-\lambda) \beta\right) \in G_{f}, \quad \forall \lambda \in\left[0, d_{\eta}(x, y)\right]
$$

Therefore, $G_{f}$ is a geodesic locally starshaped invex set corresponding to $M$. From Theorem 3.3, it follows that $f$ is geodesic semilocal preinvex on $S$ with respect to $\eta$.

\section{Conclusion}

In this paper, we introduced two new classes of functions called geodesic semilocal preinvex functions and semistrictly geodesic semilocal preinvex functions on Riemannian manifolds. We have shown that for semistrictly geodesic semilocal preinvex function, a local minimum is a global one. Next, we proved that the intersection of a family of geodesic locally starshaped $G$-invex sets is geodesic locally starshaped $G$-invex set and intersection of geodesic locally starshaped invex sets is geodesic locally starshaped invex set. We have established that the linear combination of geodesic semilocal preinvex functions is also geodesic semilocal preinvex function. After this, we established a relation between geodesic semilocal preinvex functions and its lower section of the function. Finally, we characterize some properties of geodesic semilocal preinvex functions in terms of their epigraphs. The results of the paper generalizes and extends some earlier results from Agarwal et al. [1], Yang and Li [32], Upadhyay et al. [29] and references therein. The results of this paper and the results of Barani and Puryayewali [4] could be used to establish necessary and sufficient optimality conditions for vector optimization problems on Riemanninan manifols. This will be our course of study in subsequent work.

Acknowledgements: I am very grateful to Prof. S.K. Mishra, Department of Mathematics, Institute of Science, BHU, Varanasi for guidance and valuable suggestions. I have completed this work at DST-CIMS, BHU, Varanasi, U.P., India. 


\section{References}

[1] Agarwal, R.P., Ahmad, I., Iqbal, A., Ali, S.: Geodesic G-invex sets and semistrictly geodesic $\eta$-preinvex functions. Optimization: A J. of Math. Prog. and Operations Research 61, 1169-1174 (2012)

[2] Barani, A., Pouryayevali, M.R.: Invex sets and preinvex functions on Riemannian manifolds. J. Math. Anal. Appl. 328 767-779 (2007)

[3] Barani, A., Pouryayevali, M.R.: Invariant monotone vector fields on Riemannian manifolds. Nonlinear Anal.: Theory Methods Appl. 70 1850-1861 (2009)

[4] Barani, A., Pouryayevali, M.R.: Vector Optimization problems under $d$ invexity on Riemannian manifolds. Differential Geometry Dynamical System 13 34-44 (2011)

[5] Ben-Israel, A., Mond, B.; What is invexity?. J. Aust. Math. Soci., Series B 28 1-9 (1986)

[6] Craven, B.D.: Duality for Generalized Convex Fractional Programs in Generalized concavity in optimization and Economic. Academic Press, New York (1981)

[7] Ewing, G. M.: Sufficient conditions for global minima of suitably convex functionals from variational and control theory. SIAM Review 19(2) 202$220(1977)$

[8] Hanson, M.A.: On sufficiency of the KuhnTucker conditions. J. Math. Anal. Appl. 80 545-550 (1981)

[9] Kaul, R. N., Kaur, S.: Generalizations of convex and related functions. European Journal of Operational Research 9(4) 369-377 (1982)

[10] Kaul, R. N., Kaur, S.: Sufficient optimality conditions using generalized convex functions. Opsearch 19(4) 212-224 (1982)

[11] Kaur, S.: Theoretical studies in mathematical programming. Ph.D. Thesis, University of Delhi, New Delhi, India (1983)

[12] Klingenberg, W.: Riemannian geometry. Walter de Gruyter Studies in Mathematics, Walter de Gruyter, Berlin (1982)

[13] Lang, S.: Fundamentals of Differential Geometry. Graduate Texts in Mathematics, Springer-Verlag, New York (1999)

[14] Li, C., Mordukhovich, B.S., Wang, J., Yao, J.C.: Weak sharp minima of Riemannian manifolds. SIAM J. Optim. 21 1523-1560 (2011)

[15] Mangasarian, O.L.: Nonlinear Programming. McGraw Hill Book Company, New York (1969)

[16] Mishra, S.K., Wang, S.Y., Lai, K.K.: Multiple objective fractional programming involving semilocally type I-preinvex and related functions. Journal of Mathematical Analysis and Applications 310(2) 626-640 (2005) 
[17] Mishra, S.K., Upadhyay, B.B.: Duality in nonsmooth multiobjective fractional programming involving $\eta$-pseudolinear functions. Ind. J. Indust. Appl. Math. 3 152-161 (2012)

[18] Mishra, S.K., Upadhyay, B.B.: Efficiency and duality in nonsmooth multiobjective fractional programming involving $\eta$-pseudolinear functions. Yugoslav J. Oper. Res. 22 3-18 (2012)

[19] Mishra, S.K., Upadhyay, B.B.: Nonsmooth minimax fractional programming involving $\eta$-pseudolinear functions. Optimization 63 775-788 (2014)

[20] Mishra, S.K., Upadhyay, B.B.: Pseudolinear Functions and Optimization. CRC Press (2014)

[21] Mishra, S.K., Upadhyay, B.B., Hoai An, L.T.: Lagrange multiplier characterizations of solution sets of constrained nonsmooth pseudolinear optimization problems. J. Optim. Theory Appl. 160 763-777 (2014)

[22] Mishra, S.K., Wang, S.Y., Lai K.K.: Generalized Convexity and Vector Optimization. Springer-Verlag, Berlin, Heidelberg (2009)

[23] Mititelu, S.: Generalized invexity and vector optimization on differentiable manifolds. Differ. Geom. Dyn. Syst. 3 21-31 (2001)

[24] Pini, R.: Convexity along curves and invexity. Optimization 29 301-309 (1994)

[25] Preda, V., Stancu-Minasian, I.M., Batatorescu, A.: Optimality and duality in nonlinear programming involving semilocally preinvex and related functions. Journal of Information \& Optimization Sciences 17(3) 585-596 (1996)

[26] Preda, V., Stancu-Minasian, I.M.: Duality in multiple objective programming involving semilocally preinvex and related functions. Glasnik Matematicki. Serija III 32(1) 153-165 (1997)

[27] Rapcsak, T.: Smooth Nonlinear Optimization in $\mathbb{R}^{n}$. Kluwer Academic Publishers, Dordrecht (1997)

[28] Udriste, C.: Convex Functions and Optimization Methods on Riemannian Manifolds. Kluwer Academic Publishers, Dordrecht, Netherlands (1994)

[29] Upadhyay, B.B., Mishra, S.K., Porwal, S.K.: Explicitly geodesic bpreinvex functions on Riemannian manifolds. Trans. Math. Program. Appl. 3(2) 1-14 (2015)

[30] Weir, T., Jeyakumar, V.: A class of nonconvex functions and mathematical programming. Bull. Aust. Math. Soc. 38 177-189 (1988)

[31] Weir, T., Mond, B.: Preinvex functions in multiobjective optimization. J. Math. Anal. Appl. 136 29-38 (1988)

[32] Yang, X.M., Li, D.: Semistrictly preinvex functions. J. Math. Anal. Appl. 258 287-308 (2001) 